\title{
Fasciola hepatica y otras parasitosis gastrointestinales en bovinos de doble propósito del municipio Sabana de Torres, Santander, Colombia
}

\section{Survey of Fasciola hepatica and other gastrointestinal parasitosis in dual-purpose cattle of the Sabana de Torres municipality, Santander, Colombia}

\author{
Juan Carlos Pinilla ${ }^{1,3}$, Nelson Uribe Delgado ${ }^{2}$, Angel Alberto Florez ${ }^{1}$
}

\section{RESUMEN}

\begin{abstract}
El propósito de esta investigación fue determinar la prevalencia de Fasciola hepatica y otras parasitosis gastrointestinales en bovinos doble propósito del municipio Sabana de Torres, departamento de Santander, Colombia. Se colectaron 196 muestras fecales y de sangre en 18 fincas de doble propósito. Los animales fueron estratificados en tres grupos etarios: 0-12, 12-24 y >24 meses. Las muestras fecales se procesaron mediante las técnicas coprológicas de McMaster y Dennis, y la presencia de anticuerpos anti-F. hepatica se determinó mediante una prueba de ELISA indirecto. Se identificaron los géneros de los parásitos según tamaño y morfología de sus huevos o de las larvas infectivas. La prevalencia global de parásitos gastrointestinales fue de $36.7 \%$, siendo los valores más altos para Eimeria sp (19.4\%) y Paramphistomum sp (9.2\%). La prevalencia coprológica y serológica de $F$. hepatica fue de 4.1 y $6.1 \%$, respectivamente, lo que refleja que el municipio Sabana de Torres es una zona hipoendémica para $F$. hepatica. Se encontró asociación estadística $(\mathrm{p}<0.05)$ entre el parasitismo por Eimeria sp y el grupo etario.
\end{abstract}

Palabras clave: bovinos; Fasciola; gastrointestinal; parásitos

\section{Abstract}

The aim of this research was to determine the prevalence of Fasciola hepatica and other gastrointestinal parasites in dual-purpose cattle from the municipality Sabana de Torres, department of Santander, Colombia. Faecal and blood samples $(n=196)$ were collected in 18 dual-purpose farms. The animals were stratified into three age groups: 0-

\footnotetext{
${ }^{1}$ Universidad de Santander, Facultad de Ciencias Exactas, Naturales y Agropecuarias, Grupo de Investigación en Ciencia Animal-GICA, Bucaramanga, Colombia

${ }^{2}$ Universidad Industrial de Santander, Facultad de Salud, Grupo de Investigación en Epidemiología Molecular-GIEM, Bucaramanga, Colombia

${ }^{3}$ E-mail: jcpinilla@hotmail.com
}

Recibido: 23 de enero de 2019

Aceptado para publicación: 5 de agosto de 2019 
12, 12-24 and >24 months. Faecal samples were processed by the McMaster and Dennis coprological techniques, and the presence of antibodies anti-F. hepatica was determined by an indirect ELISA test. The genera of the parasites were identified according to the size and morphology of their eggs or infective larvae. The overall prevalence of gastrointestinal parasites was 36.7\%, with the highest values for Eimeria sp (19.4\%) and Paramphistomum sp (9.2\%). The coprological and serological prevalence of $F$. hepatica was 4.1 and $6.1 \%$, respectively, which reflects that the municipality Sabana de Torres is a hypoendemic zone for $F$. hepatica. A statistical association $(\mathrm{p}<0.05)$ was found between Eimeria sp parasitism and the age group.

Key words: cattle; Fasciola; gastrointestinal parasites

\section{INTRODUCCIÓN}

Las parasitosis gastrointestinales (PGI) son uno de los problemas sanitarios más importantes en el ganado bovino, ya que ocasionan pérdidas económicas por disminución en la producción de leche y carne, e incremento en los costos asociados al tratamiento y control (Cordero del Campillo et al., 1999). La mayor parte de las PGI en el bovino son ocasionadas por protozoarios, nematodos y trematodos. Dentro de los protozoarios, Eimeria sp es un enterococcidio que afecta clínicamente animales menores de un año e inmunosuprimidos (Shepelo et al., 2015). Los nematodos ocasionan problemas de gastroenteritis caracterizadas clínicamente por diarrea, debilidad, hemorragias y deshidratación (Mawatari et al., 2014). Los géneros de mayor importancia y prevalentes a nivel mundial pertenecen a las familias Trichostrongylidae, Ancylostomidae, Ascarididae, Strongyloididae y Trichuridae (Colina et al., 2013).

Fasciola hepatica es un parásito de la clase Trematoda, subclase Digenea, de distribución mundial (Cordero del Campillo et al., 1999), que afecta a los bovinos y a gran cantidad de mamíferos (Brockwell et al., 2014), incluyendo al hombre. En Colombia, esta parasitosis ocasiona pérdidas económicas anuales estimadas en \$12 483 millones de pesos colombianos (Becerra, 2001; Pulido et al., 2010; Bernardo et al., 2011), debi- das al decomiso de hígados (9.18\%), disminución en la producción de leche y de la fertilidad de las vacas (Valencia et al., 2012). Paramphistomum sp es un paramfistomidio de importancia veterinaria, responsable de la paramfistomiasis en bovinos, búfalos, camélidos, ovinos y caprinos (Muro y Ramajo, 2002).

Con relación a reportes de prevalencia parasitaria, Chaparro et al. (2016) señalaron $36.7 \%$ de prevalencia de coccidias en bovinos lecheros de Antioquia, Colombia, mientras que Rodríguez-Vivas et al. (2001) reportaron $72 \%$ de prevalencia en bovinos del estado de Yucatán, México. En el grupo de nematodos, la literatura es abundante en reportes de prevalencia (Orjuela et al., 1991; Rodríguez-Vivas et al., 2001; Urdaneta et al., 2011; Colina et al., 2013). Con respecto a $F$. hepatica, Ticona et al. (2010) señalaron 95.5\% de seroprevalencia en bovinos del Perú, mientras que en Cuba se reportaron prevalencias entre 58.3 y $62.5 \%$ en animales de doble propósito (Soca-Pérez et al., 2016). En Mérida, Venezuela, se reportó $23.8 \%$ de prevalencia por coprología en ganado lechero (Gauta et al., 2011) y de $7.3 \%$ de seropositividad en el estado Zulia (AnguloCubillán et al., 2013). La mayor prevalencia de fascioliosis bovina en Colombia ha sido señalada en las zonas ganaderas de climas fríos, donde es endémica y afecta al $25 \%$ del ganado lechero (Estrada et al., 2006), con rangos de prevalencia entre 25 y $80 \%$ (Becerra, 2001); sin embargo, también ha sido señalado en regiones bajas de Colombia 
(Bedoya et al., 2012; Recalde-Reyes et al., 2014; Giraldo et al., 2016).

El municipio Sabana de Torres se encuentra ubicado en el nororiente colombiano. Es una región agropecuaria, siendo el sector ganadero uno de los más importantes en la economía regional, con una participación del $30 \%$ del inventario vacuno a nivel regional. Esta región se destaca por su industria láctea; sin embargo, cerca del $75 \%$ de las explotaciones ganaderas están orientadas al doble propósito (carne y leche). Según cifras del Instituto Colombiano Agropecuario (ICA, 2017), el municipio cuenta con una población bovina de 110000 animales. En esta región no se han llevado a cabo estudios sobre la prevalencia de $F$. hepatica y parasitismo gastrointestinal en bovinos; sin embargo, los productores de la zona manifiestan conocer el impacto económico de las parasitosis. Además, se han presentado evidencias del trematodo en centrales de sacrificio en informes no oficiales. Por lo tanto, se planteó como objetivo de esta investigación determinar la prevalencia de Fasciola hepatica y otras parasitosis gastrointestinales en bovinos del municipio Sabana de Torres, departamento Santander, Colombia.

\section{Materiales y MéTodos}

\section{Ubicación Geográfica}

El estudio se realizó en el municipio Sabana de Torres $\left(7^{\circ} 232253 \mathrm{~N} 73^{\circ} 302013 \mathrm{O}\right)$ del departamento del Santander. La región presenta una temperatura media anual de $28^{\circ} \mathrm{C}$, una altitud de $110 \mathrm{msnm}$ y una precipitación media anual de $1750 \mathrm{~mm}$, con dos periodos de lluvias bien definidos al año.

\section{Diseño del Muestreo}

Se diseñó un muestreo por conveniencia, de tipo descriptivo y corte transversal. Entre enero y marzo de 2018 (verano) se muestrearon 18 fincas ubicadas en el muni- cipio Sabana de Torres, donde el promedio de animales por finca era de 250 bovinos. Según los registros de vacunación del ICA (2017), la población censada del municipio fue de 110000 animales, y empleando la fórmula para poblaciones conocidas (Thrusfield, 2007), con una prevalencia esperada de $25 \%$ (Estrada et al., 2006) y un nivel de confianza de $90 \%$ y un error máximo asociado (EMA) de $6 \%$, se determinó el requerimiento de 196 animales. Este número fue distribuido proporcionalmente según la cantidad de animales de las fincas.

Los animales seleccionados eran de tipo doble propósito, entre 6 meses y 8 años, y se tomaron a criterio del productor. Se formaron tres grupos etarios: $0-12,12-24 \mathrm{y}>24$ meses. Se tomaron muestras de heces y de sangre de cada animal. La toma de la muestra fecal $(10 \mathrm{~g})$ se realizó por vía rectal, utilizando bolsas de polietileno, y enviadas refrigeradas al Laboratorio de Parasitología de la Universidad de Santander para su procesamiento al cabo de tres días. Las muestras de sangre $(5 \mathrm{ml})$ se tomaron directamente de la vena coccígea, utilizando tubos Vacutainer ${ }^{\circledR}$ sin anticoagulante; se centrifugaron a $5600 \mathrm{~g}$ por $10 \mathrm{~min}$ para la obtención de suero, los cuales se mantuvieron a $-21{ }^{\circ} \mathrm{C}$ hasta su procesamiento en el laboratorio de Parasitología. En una planilla de campo se registró la especie, raza, sexo, edad y condición corporal de los animales muestreados.

\section{Procedimientos de Diagnóstico}

Las muestras fecales se procesaron mediante dos técnicas coproparasitológicas:

- Técnica de Sedimentación de Dennis modificada (Correa et al., 2016) para la determinación de huevos pesados de $F$. hepatica y Paramphistomum sp.

- Técnica de McMaster para detección de ooquistes de coccidias (opg) y huevos de nematodos (hpg), empleando una solución de flotación de azúcar-sal $(1 \mathrm{~L}$ de solución saturada de $\mathrm{NaCl}$ más 500 g de 
azúcar) (Henriksen y Christensen, 1992; Sandoval et al., 2011).

Las especies de trematodos y géneros de nematodos fueron identificados por la morfología y tamaño de sus huevos (Fiel et al., 2011), empleando una lupa estereoscópica de luz con magnificación de 10 y 40x. La diferencia entre huevos de $F$. hepatica y Paramphistomun sp se realizó por el color.

Además, se determinó la presencia de anticuerpos anti-F. hepatica mediante un kit comercial para el ensayo de inmunoabsorción enzimático de ELISA indirecto (Bio-X Diagnostics Laboratory). Para la interpretación de los resultados se empleó un valor porcentual de densidad óptica, que permitió clasificar los resultados en negativos y positivos. El procedimiento se realizó de acuerdo con las instrucciones del fabricante.

\section{Análisis Estadístico}

La prevalencia se determinó dividiendo el número de animales positivos entre el total de animales de la población muestreada. Los resultados obtenidos se analizaron mediante estadísticos descriptivos y test de Chi-cuadrado para determinar asociaciones estadísticas. Para los cálculos se utilizó el programa estadístico SPSS v. 21.

\section{Resultados y Discusión}

La prevalencia global del PGI fue en el $100 \%$ de las fincas (18/18), mientras que la prevalencia individual fue de $36.7 \%$ (72/196); resultado que coincide con Chaparro et al. (2016), en ganado lechero del departamento de Antioquia, Colombia. Sin embargo, otros autores señalan mayores valores de prevalencia $(>85 \%)$ en ganado vacuno mantenido en condiciones climáticas semejantes a las encontradas en el presente estudio (Urdaneta et al., 2011; Colina et al., 2013; Pinilla et al., 2018). Por otro lado, la tasa de prevalencia global en los meses de verano (enero y mar-
Cuadro 1. Prevalencia de géneros parasitarios diagnosticados en 196 bovinos del municipio Sabana de Torres, departamento Santander (Colombia)

\begin{tabular}{lcc}
\hline Parásito & $\begin{array}{c}\text { Positivos } \\
(\mathrm{n})\end{array}$ & $\begin{array}{c}\text { Prevalencia } \\
(\%)\end{array}$ \\
\hline Eimeria $\mathrm{sp}$ & 38 & 19.4 \\
Paramphistomum $\mathrm{sp}^{1}$ & 18 & 9.2 \\
Strongylida $^{1}$ & 12 & 6.1 \\
Fasciola hepatica $^{\text {Strongyloides } \mathrm{sp}}$ & 8 & 4.1 \\
\hline
\end{tabular}

${ }^{1}$ Se agrupó como sub-orden Strongylida

zo) estuvo notablemente baja, debido probablemente a que las condiciones climatológicas (temperatura y humedad) durante esos meses no favorecen el mantenimiento de las formas infectantes de la mayoría de géneros parasitarios (Cordero del Campillo et al., 1999).

En el Cuadro 1 se muestra un análisis descriptivo de los cinco géneros/especies parasitarios encontrados en el estudio, siendo Eimeria sp (19.4\%) el más prevalente, seguido de Paramphistomum sp $(9.2 \%)$ y parásitos del sub-orden Strongylida (6.1\%). Así mismo, F. hepatica mostró una prevalencia coprológica de $4.1 \%$. La prevalencia de protozoarios del género Eimeria coincide con lo reportado por Chaparro et al. (2016) y Pinilla et al. (2019); sin embargo, resultados difieren con lo demostrado por Díaz de Ramírez et al. (1998) y Rodríguez-Vivas et al. (2001), quienes reportaron prevalencias de 53 y $71.5 \%$ de coccidias en bovinos de México. Es posible que la baja prevalencia del género Eimeria se encuentre asociada con la toma de muestras en el periodo seco del año. Rodríguez-Vivas et al. (2001) encontraron en el trópico mexicano que uno de los factores asociados a la mayor excreción de ooquistes de Eimeria en el ganado bovino era la época de lluvias. 
Con respecto al grupo de nematodos, los parásitos agrupados en el sub-orden Strongylida mostraron una prevalencia más baja con respecto al $60.6 \%$ reportado por Rodríguez-Vivas et al. (2001). Si bien, en este estudio no se identificaron géneros ni especies, el género Haemonchus ha sido reportado con mayor frecuencia (Rodríguez-Vivas et al., 2001).

En el caso de Trematodos, la prevalencia de $4.1 \%$ de $F$. hepatica coincide con los datos señalados por Recalde-Reyes et al. (2014) y Pinilla et al. (2018) en bovinos de los departamentos del Quindio y Cesar, Colombia, respectivamente, y con Rojas y Cartín (2016) en bovinos de Costa Rica. Sin embargo, los resultados del presente estudio difieren con lo reportado por Soca-Pérez et al. (2016), Chaparro et al. (2016) y Pinilla et al. (2019) quienes señalaron 58.3, 30.9 y 22.3\% de prevalencia en Cuba, en Antioquia (Colombia) y en las montañas del Nororiente de Colombia, respectivamente. Aunque no existen antecedentes de estudios sobre prevalencia de este trematodo y decomisos de hígado de animales provenientes de la zona en estudio, la baja prevalencia de $F$. hepatica podría deberse a la escasa interacción entre los animales susceptibles y los hospederos intermediarios (Rojas y Cartín, 2016). Por otro lado, en Colombia se han registrado trematodos de los géneros Paramphistomum y Cotylophorum en bovinos de la Costa Caribe y Llanos Orientales (Parra et al., 1982) y en fincas de Río Negro, Antioquia (Alarcón y Velásquez, 2009). La semejanza en las características morfológicas de las especies de paramfistómidos, así como la falta de experiencia en la identificación de estas especies se convierte en un obstáculo para el desarrollo de estudios epidemiológicos y de prevalencia, por lo que se propone incluir el uso de secuencias genómicas (López y Velásquez, 2009).

En el Cuadro 2 se muestra la comparación entre los porcentajes de positividad de la infección por diferentes géneros parasita- rios con respecto al grupo etario. En el parasitismo por Eimeria sp los animales de 0-12 meses mostraron la mayor prevalencia (57.1\%), y los mayores de 24 meses la menor prevalencia $(12.3 \%)(\mathrm{p}<0.05)$. Por otro lado, no se encontró asociación estadística entre la infección por parásitos del sub-orden Strongylida, y Strongyloides sp y Paramphistomum sp con respecto a la edad de los animales.

La mayor prevalencia de Eimeria sp en animales jóvenes ( $0-12$ meses) coincide con los hallazgos reportados por otros autores (Díaz de Ramírez et al., 1998; Tomczuk et al., 2015; Chaparro et al., 2016) que señalan mayor excreción de ooquistes en animales jóvenes, ya que la mayoría de especies de Eimeria de bovinos tienen periodos prepatentes de 2 y 3 semanas, de modo que los becerros pueden ingerir una cantidad suficiente de ooquistes esporulados para establecer una infección patente en el rebaño (Díaz de Ramírez et al., 1998). Los terneros en los pastizales generalmente albergan mayores cargas de coccidios que los adultos (Pfukenyi et al., 2007), dado que inmunológicamente son menos capaces de prevenir el establecimiento de parásitos. En este estudio, los bovinos adultos se podrían comportar como portadores asintomáticos y potenciales fuentes de infección de coccidios para los becerros (Quiroz et al., 2011).

En el Cuadro 3 se muestra la comparación entre los porcentajes de positividad de la infección por $F$. hepatica según las técnicas de Dennis y ELISA con respecto a los grupos etarios. Los animales menores de un año no mostraron evidencia de la infección, pero aun así no se encontró asociación estadística con respecto a la edad de los animales. La relativa mayor prevalencia de $F$. hepatica en animales mayores de 12 meses se puede explicar por el hecho de un mayor tiempo de exposición a la infección (Yasin et al., 2018). Por otro lado, el ciclo de vida de este parásito requiere entre 2 y 3 meses para el desarrollo de las larvas (Chaparro et al., 2016). 
Cuadro 2. Comparación entre la prevalencia (\%) de parasitismo por Eimeria sp, orden Strongylida, Strongyloides sp y Paramphistomum sp con el grupo etario en bovinos del municipio Sabana de Torres, Santander (Colombia)

\begin{tabular}{cccccc}
\hline $\begin{array}{c}\text { Grupo etario } \\
(\mathrm{meses})\end{array}$ & $\begin{array}{c}\text { Bovinos } \\
(\mathrm{n})\end{array}$ & $\begin{array}{c}\text { Eimeria } \\
\mathrm{sp}(\%)\end{array}$ & $\begin{array}{c}\text { Strongylida } \\
(\%)\end{array}$ & $\begin{array}{c}\text { Strongyloides } \\
\mathrm{sp}(\%)\end{array}$ & $\begin{array}{c}\text { Paramphistomum } \\
\mathrm{sp}(\%)\end{array}$ \\
\hline $0-12$ & 14 & 57.1 & 14.3 & 0 & 0 \\
$12-24$ & 52 & 26.9 & 7.7 & 3.8 & 7.7 \\
$>24$ & 130 & 12.3 & 4.6 & 0 & 10.8 \\
\hline Total & 196 & 19.4 & 6.1 & 1 & 9.2 \\
\hline \multirow{2}{*}{ Chi-cuadrado } & & {$\left[\mathrm{X}^{2}=18.8 ;\right.$} & {$\left[\mathrm{X}^{2}=2.3 ;\right.$} & {$\left[\mathrm{X}^{2}=5.6 ;\right.$} & {$\left[\mathrm{X}^{2}=1.9 ;\right.$} \\
& $\mathrm{p}=0.00]$ & $\mathrm{p}=0.31]$ & $\mathrm{p}=0.06]$ & $\mathrm{p}=0.37]$ \\
\hline
\end{tabular}

${ }^{1}$ Se agrupó como sub-orden Strongylida

Cuadro 3. Comparación entre la prevalencia (\%) de parasitismo por Fasciola hepatica en bovinos, de acuerdo con los resultados de la prueba de Dennis y test de ELISA, según el grupo etario en bovinos del municipio Sabana de Torres, Santander (Colombia)

\begin{tabular}{cccccc}
\hline \multirow{2}{*}{ Grupo etario (meses) } & \multirow{2}{*}{ Bovinos (n) } & \multicolumn{2}{c}{ Positivos a Dennis } & \multicolumn{2}{c}{ Positivos a ELISA } \\
\cline { 3 - 6 } & & $\mathrm{n}$ & $\%$ & $\mathrm{n}$ & $\%$ \\
\hline $0-12$ & 14 & 0 & 0 & 0 & 0 \\
$12-24$ & 52 & 2 & 3.8 & 4 & 7.7 \\
$>24$ & 130 & 6 & 4.6 & 6 & 4.6 \\
\hline Total & 196 & 8 & 4.1 & 10 & 5.1 \\
\hline Chi cuadrado & & {$\left[\mathrm{X}^{2}=0.69 ; \mathrm{p}=0.7\right]$} & {$\left[\mathrm{X}^{2}=1.53 ; \mathrm{p}=0.47\right]$} \\
\hline
\end{tabular}

De acuerdo con el $5.1 \%$ de seroprevalencia de $F$. hepatica en el presente estudio y empleando la clasificación señalada por Valderrama (2016), el municipio Sabana de Torres es una zona hipoendémica $(<10 \%$ de seroprevalencia) de F. hepatica. No obstante, las características de la región estudiada son favorables para la presencia de caracoles Limneidos y el desarrollo de $F$. hepatica.

\section{Conclusiones}

Los bovinos del municipio Sabana de Torres, Santander, Colombia, se encuentran infectados con parásitos gastrointestinales de los géneros Eimeria, Paramphistomum y Fasciola.

\section{Literatura Citada}

1. Alarcón EP, Velásquez LE. 2009. Descripción morfológica de Cotylophoron cotylophorum (Digenea: Paramphistomidae) hallado en bovinos de Rio negro, Antioquia, Colombia. Rev Colomb Cienc Pec 22: 168-177.

2. Angulo-Cubillán F, Chacín E, Sánchez $A$, Calle M, Zambrano S, Montero M, Pérez M, et al. 2013. Detección de anticuerpos IgG frente a Fasciola 
hepatica en un rebaño criollo limonero del municipio Mara, estado Zulia, Venezuela. Rev Cient Fac Cien V 22: 471-474.

3. Becerra W. 2001. Consideraciones sobre estrategias sostenibles para el control de Fasciola hepatica en Latinoamérica. Rev Colomb Cienc Pec 14: 28-35.

4. Bedoya J, Hurtado Y, Pérez J, Solano S, Úsuga V, Vanegas M, Gómez C, et al. 2012. Primer registro de focos de fasciolosis y paramfistomosis en bovinos doble propósito, Gómez Plata, Antioquia, Colombia. Hechos Microbiol 3: 31-39.

5. Bernardo CD, Carneiro MB, Avelar BR, Donatele DM, Mar-Tins IV, Pereira MJ. 2011. Prevalence of liver condemnation due to bovine fasciolosis in Southern Espírito Santo: temporal distribution and economic losses. Rev Bras Parasitol V 20: 49-53.

6. Brockwell YM, Elliott TP, Anderson GR, Stanton R, Spithill TW, Sangster NC. 2014. Confirmation of Fasciola hepatica resistant to triclabendazole in naturally infected Australian beef and dairy cattle. Int J Parasitol Drugs Drug Resist 4: 48-54. doi: 10.1016/j.ijpddr.2013.11.005

7. Chaparro JJ, Ramírez NF, Villar D, Fernandez JA, Londoño J, Arbeláez C, López L, et al. 2016. Survey of gastrointestinal parasites, liver flukes and lungworm in feces from dairy cattle in the high tropics of Antioquia, Colombia. Parasite Epidemiol Contr 1: 124-130. doi: 10.1016/j.parepi.2016.05.001

8. Colina JC, Mendoza GA, Jara CA. 2013. Prevalencia e intensidad del parasitismo gastrointestinal por nematodos en bovinos, Bos taurus, del distrito Pacanga (La Libertad, Perú). Rebiol 33: 76-83.

9. Cordero del Campillo M, Rojo F, Martínez A, Sánchez C, Hernández S, Navarrete J, Díez P, et al. 1999. Parasitología veterinaria. Madrid, España: McGraw Hill Interamericana. $935 \mathrm{p}$.

10. Correa S, Martínez, Y, López J, Velásquez L. 2016. Evaluación de la técnica modificada de Dennis para el diagnóstico de fasciolosis bovina. Biomed 36:
64-68. doi: 10.7705/biomedica.v36i2.2875

11. Díaz de Ramírez A, Justo JA, González M, Piña EF, Ramírez LI. 1998. Prevalencia de coccidios en bovinos de Los Llanos de Monay, estado Trujillo, Venezuela. Rev Cient Fac Cien V 8: 346-353.

12. Estrada VE, Gómez M, Velásquez LE. 2006. La higiene del ganado y la fasciolosis bovina, Medellín y Rionegro, 1914-1970. IATREIA 19: 393-407.

13. Fiel C, Steffan P, Ferreyra P. 2011. Diagnóstico más frecuente de las parasitosis de los rumiantes: técnicas de laboratorio e interpretación de resultados. Buenos Aires, Argentina: Abad Benjamin. $131 \mathrm{p}$.

14. Gauta J, Pérez A, Lecuna J, García $M$, Aguirre A, Armas S, Quijada J. 2011. Prevalencia de Fasciola hepatica en ganadería de altura en Bailadores Mérida, Venezuela. REDVET 12(11). [Internet]. Disponible en: https:// recyt.fecyt.es/index.php/REDVET/ article/view/15208

15. Giraldo E, Pérez J, Aguilar S, Linares S. 2016. Prevalencia de fasciolosis bovina en una zona de Caldas, Colombia, con evidencias de la enfermedad. Rev UDCA Act Div Cient 19: 139-148.

16. Henriksen SA, Christensen JP. 1992. Demonstration of Isospora suis oocysts in faecal samples. Vet Rec 131: 443-444. doi: $10.1136 /$ vr.131.19.443

17. [ICA] Instituto Colombiano Agropecuario. 2017. Censo Pecuario Nacional. [Internet]. Disponible en: https:// www.ica.gov.co/Areas/Pecuaria/Servicios/Epidemiologia-Veterinaria/Censos2016/Censo-2017.aspx

18. López J, Velásquez L. 2009. Cotylophoron panamensis (Digenea: Paramphistomidae) en bovinos del Meta y del Guaviare, Colombia. Acta Biol Colomb 17: 421-430.

19. Mawatari T, Hirano K, Ikeda H, Tsunemitsu H, Suzuki T. 2014. Surveillance of diarrhea-causing pathogens in dairy and beef cows in Yamagata 
Prefecture, Japan from 2002 to 2011. Microbiol Immunol 58: 530-535. doi: 10.1111/1348-0421.12174

20. Muro A, Ramajo M. 2002. Paramfistomosis. In: Cordero del Campillo, Rojo $\mathrm{F}$ (eds). Parasitología veterinaria. España: McGraw-Hill. p 225-228.

21. Orjuela J, Navarrete M, Betancourt A, Roqueme L, Cortez E, Morrison R. 1991. Salud y productividad en bovinos de la costa norte de Colombia. World Anim Rev 69: 7-14.

22. Parra D, Gallego M, Griffiths I. 1982. Prevalencia de la paramfistomiasis bovina en hatos lecheros de Colombia. Rev Colomb Cienc Pec 982: 33-34.

23. Pfukenyi DM, Mukaratirwa S, Willingham AL, Monrad J. 2007. Epidemiological studies of parasitic gastrointestinal nematodes, cestodes and coccidian infections in cattle in the Highveld and lowveld communal grazing areas of Zimbabwe. Onderstepoort J Vet 74: 129-142. doi: 10.4102/ojvr.v74i2.132

24. Pinilla JC, Flórez P, Sierra M, Morales E, Sierra R, Vásquez MC, Tobón $J C$, et al. 2018. Prevalencia del parasitismo gastrointestinal en bovinos del departamento Cesar, Colombia. Rev Inv Vet Perú 29: 278-287. doi: 10.15381/ rivep.v29i1.14202

25. Pinilla León JC, Delgado NU, Florez $A A$. 2019. Prevalence of gastrointestinal parasites in cattle and sheep in three municipalities in the Colombian Northeastern Mountain. Vet World 12: 4854. doi: 10.14202/vetworld.2019.48-54

26. Pulido A, Castañeda R, Arbeláez G. 2010. Fasciola hepatica: pedagogía de diagnóstico por laboratorio y su situación en Colombia. REDVET 12(5B).

27. Quiroz H, Figueroa J, Ibarra F, López M. 2011. Epidemiología de enfermedades parasitarias en animales domésticos. México. $655 \mathrm{p}$.

28. Recalde-Reyes D, Padilla L, Giraldo M, Toro L, González M, Castaño J. 2014. Prevalencia de Fasciola hepatica en humanos y bovinos en el depar- tamento del Quindío-Colombia 20122013. Infectio 18:153-157. doi: 10.1016/ j.infect.2014.09.001

29. Rodríguez-Vivas RI, Cob-Galera LA, Domínguez-Alpizar JL. 2001. Frecuencia de parásitos gastrointestinales en animales domésticos diagnosticados en Yucatán, México. Biomédica 12: 19-25.

30. Rojas D, Cartín JA. 2016. Prevalencia de Fasciola hepatica y pérdidas económicas asociadas al decomiso de hígados en tres mataderos de clase A de Costa Rica. Agron Costarric 40: 53-62.

31. Sandoval E, Morales G Ybarra N, Barrios M, Borges J. 2011. Comparación entre dos modelos diferentes de cámaras de McMaster empleadas para el conteo coproscópico en el diagnóstico de infecciones por nematodos gastroen-téricos en rumiantes. Zootecnia Trop 29: 495-501.

32. Shepelo P, Gitau GK, Mulei CM, Vanleeuwen J, Richards S, Wichtel J, Uehlinger F et al. 2015. Prevalence of Cryptosporidia, Eimeria, Giardia, and Strongyloides in pre-weaned calves on smallholder dairy farms in Mukurwe-ini district, Kenya. Vet World 8: 1118-1125. doi: 10.14202/vetworld.2015.1118-1125

33. Soca-Pérez M, Giupponi-Cardoso P, López-Vigoa $O$, Sanavria A, SánchezSantana T, Labrada-Vázquez A. 2016. Prevalencia de Fasciola hepatica en vacas en pastoreo durante el periodo poco lluvioso. Pastos y Forrajes 39: 281-285.

34. Thrusfield M. 2007. Veterinary epidemiology. $3^{\text {rd }}$ ed. UK: Blackwell. $624 \mathrm{p}$.

35. Ticona D, Chávez A, Casas G, Chavera CA, Li E. 2010. Prevalencia de Fasciola hepatica en bovinos y ovinos de Vilcashuamán, Ayacucho. Rev Inv Vet Perú 21: 168-174.

36. Tomczuk K, Grzybek M, Szczepaniak K, Studziñska M, Demkowska M, Roczen M, Klockiewicz M. 2015. Analysis of intrinsic and extrinsic factors influencing the dynamics of bovine Eimeria spp from central-eastern Poland. Vet Parasitol 214: 22-28. doi: 10.1016/j.vetpar.2015.09.027 
37. Urdaneta M, Urdaneta A, Parra A, Chacín E, Ramírez R, Angulo F. 2011. Prevalencia y grado de infección de helmintos gastrointestinales en rebaños bovinos doble propósito del municipio Miranda del estado Zulia, Venezuela. Rev Cient Fac Cien V 2: 184-193.

38. Valderrama A. 2016. Prevalencia de fascioliasis en animales poligástricos de Perú, 1985-2015. Rev Med Vet 32: 121 129. doi: 10.19052/mv.3861

39. Valencia-López N, Malone JB, Carmona CG, Velásquez LE. 2012. Climate based risk models for Fasciola hepatica in Colombia. Geospatial Health 6: 67-85. doi: 10.4081/gh.2012.125

40. Yasin M, Alim M, Anisuzzaman, Ahasan S, Munsi M, Chowdhury E, Hatta T, Tsuji N, et al. 2018. Trematode infections in farm animals and their vector snails in Saint Martin's Island, the southeastern offshore area of Bangladesh in the Bay of Bengal. J Vet Med Sci 80: 684-688. doi: 10.1292/ jvms.17-0308 